International Journal of Pure and Applied Mathematics

Volume 110 No. 4 2016, 609-622

ISSN: 1311-8080 (printed version); ISSN: 1314-3395 (on-line version)

url: http://www.ijpam.eu

doi: 10.12732/ijpam.v110i4.4

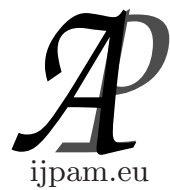

\title{
$r$-DYNAMIC CHROMATIC NUMBER OF CYCLES, COMPLETE GRAPHS AND FORESTS
}

\author{
Hermie V. Inoferio ${ }^{1}$, Michael P. Baldado Jr. ${ }^{2} \S$ \\ ${ }^{1}$ College of Education \\ Jose Rizal Memorial State University \\ Katipunan, Zamboanga del Norte, PHILIPPINES \\ ${ }^{2}$ Mathematics Department \\ Negros Oriental State University \\ Dumaguete City, Philippines
}

\begin{abstract}
Let $G=(V, E)$ be a graph. An $r$-dynamic $k$-coloring of $G$ is a function $f$ from $V$ to a set $C$ of colors such that (1) $f$ is a proper coloring, and (2) for all vertices $v$ in $V$, $|f(N(v))| \geq \min \left\{r, \operatorname{deg}_{G}(v)\right\}$. The $r$-dynamic chromatic number of a $G$, denoted by $\chi_{r}(G)$, is the smallest $k$ such that $f$ is an $r$-dynamic $k$-coloring of $G$.

This study gave the $r$-dynamic chromatic number of paths, cycles, complete graphs, empty graphs, the vertex gluing of graphs and the union of graphs. Some characterizations are also given.
\end{abstract}

AMS Subject Classification: $05 \mathrm{C} 15$

Key Words: $r$-dynamic chromatic number, $r$-dynamic $k$-coloring, cycles, vertex gluing

\section{Introduction}

As mentioned in [11], the earliest results about graph coloring is on planar graphs motivated by a problem regarding the coloring of maps. While coloring a map of the counties of England, Francis Guthrie presented the four color conjecture which stated that four colors were sufficient to color the map so that no regions sharing a common border received the same color. This idea may have motivated the concept proper vertex coloring. Using graph theoretic

Received: $\quad$ July 15, 2016

Revised: October 10, 2016

Published: November 9, 2016

$\S_{\text {Correspondence author }}$ (c) 2016 Academic Publications, Ltd.

url: www.acadpubl.eu 
parlance, a proper vertex coloring of a graph $G=(V, E)$ is a function from $V$ to a finite set $C$, whose elements are called colors, such that no two adjacent vertex are assigned the same color.

A generalization of the proper vertex coloring is the dynamic coloring of graphs. The dynamic coloring of a graph $G$ is a proper coloring such that every vertex of $G$ with degree at least two has atleast two neighbors that are colored differently. This concept was introduced by Montgomery in [3]. Since then, the concept was studied by many authors, see for example [1], [2], [4], [5], [6] and [7].

A generalization of the dynamic coloring was also introduced by Montgomery in [3]. The generalized concept is now called the $r$-dynamic $k$-coloring. The proper vertex coloring and the dynamic coloring are special cases of the concept $r$-dynamic $k$-coloring, that is, the 1 -dynamic $k$-coloring is the proper vertex coloring concept, while the 2 -dynamic $k$-coloring is the dynamic coloring concept.

The general $r$-dynamic $k$-coloring of planar grids is studied well in [8] and [9].

A vertex coloring of a graph $G=(V, E)$ is a function $f$ from $V$ to a finite set $C$, whose elements are called color. A $k$-coloring is a vertex coloring with at most $k$-colors. In this case, we always assume that $C=\{1,2, \ldots, k\}$. A $k$-coloring may also be viewed as a vertex partition $V=V_{1} \cup V_{2} \cup \cdots \cup V_{k}$ or $\left(V_{1}, V_{2}, \ldots, V_{k}\right)$, where $V_{i}=f^{-1}(i)$ are called the color classes. A graph is $k$-colorable if it admits a proper vertex coloring with at most $k$-colors. The chromatic number of a graph $G$, denoted by $\chi(G)$, is the smallest $k$ such that $G$ is $k$-colorable.

An $r$-dynamic $k$-coloring of $G$ is a proper coloring $f$ such that for all vertices $v$ in $V,|f(N(v))| \geq \min \left\{r, \operatorname{deg}_{G}(v)\right\}$. The $r$-dynamic chromatic number of a $G$, denoted by $\chi_{r}(G)$, is the smallest $k$ such that $f$ is an $r$-dynamic $k$-coloring of $G$.

Let $G_{1}, G_{2}, \ldots, G_{t}$ be disjoint graphs, each containing a complete subgraph $K_{r}(r \geq 1)$. Let $G$ be a graph obtained from the union of $t$ graphs $G_{i}$ by identifying the complete graphs $K_{r}$ (from each $G_{i}$ ) in an arbitrary way. We call $G$ a $K_{r}$-gluing of $G_{1}, G_{2}, \ldots, G_{t}$. In particular, when $r=1$ we say that $G$ is a vertex-gluing.

Hereafter, please refer to [12] for concepts that were used but were not discussed in this paper. 


\section{2. r-Dynamic Chromatic Number of Paths and Cycles}

In this section we verified the $r$-dynamic chromatic number of paths and cycles. The next Lemma, Lemma 2.1, characterizes $r$-dynamic $k$-coloring in paths.

Lemma 2.1. Let $P_{n}=\left(v_{1}, v_{2}, \ldots, v_{n}\right)$ be a path of order $n \geq 3$. Then $f: P_{n} \rightarrow\{1,2, \ldots, k\}$ is an $r$-dynamic $(r>1) k$-coloring of $P_{n}$ if and only if for all $i$ with $\operatorname{deg}\left(v_{i}\right)=2, f\left(v_{i-1}\right), f\left(v_{i}\right), f\left(v_{i+1}\right)$ are distinct.

Proof. Assume that $f$ is an $r$-dynamic $k$-coloring of $P_{n}$ and there exist $i$ such that at least two of $f\left(v_{i-1}\right), f\left(v_{i}\right), f\left(v_{i+1}\right)$ are not distinct. Consider the following cases: (Case 1. $f\left(v_{i-1}\right)=f\left(v_{i}\right)$ ) If $f\left(v_{i-1}\right)=f\left(v_{i}\right)$, then $f$ is not proper. This is a contradiction. (Case 2. $\left.f\left(v_{i-1}\right)=f\left(v_{i+1}\right)\right)$ If $f\left(v_{i-1}\right)=$ $f\left(v_{i+1}\right)$, then $f\left(N\left(v_{i}\right)\right)=f\left(\left\{v_{i-1}, v_{i+1}\right\}\right)=\left\{f\left(v_{i-1}\right)\right\}$, that is, $\left|f\left(N\left(v_{i}\right)\right)\right|=$ $\left|\left\{f\left(v_{i-1}\right)\right\}\right|=1<2=\min \{2, r\}$. This is a contradiction.

Conversely, assume that for all $i$ with $\operatorname{deg}\left(v_{i}\right)=2, f\left(v_{i-1}\right), f\left(v_{i}\right), f\left(v_{i+1}\right)$ are distinct. Let $v \in V\left(P_{n}\right)$ and consider the following cases: (Case 1. $v=v_{1}$ or $\left.v=v_{n}\right)$ If $v=v_{1}$, then $\operatorname{deg}\left(v_{1}\right)=1$. Hence, $|f(N(v))|=\left|f\left(N\left(v_{1}\right)\right)\right|=$ $\left|\left\{f\left(v_{1}\right)\right\}\right|=1=\min \{1, r\}$. (Case 2. $v \neq v_{1}$ and $v \neq v_{n}$ ) Without loss of generality, let $v=v_{2}$. Then $\operatorname{deg}(v)=\operatorname{deg}\left(v_{2}\right)=2$. Thus, $|f(N(v))|=$ $\left|f\left(N\left(v_{2}\right)\right)\right|=\left|\left\{f\left(v_{1}\right), f\left(v_{3}\right)\right\}\right|=2=\min \{2, r\}$. This shows that $f$ is an $r$-dynamic $k$-coloring of $P_{n}$.

Remark 2.2. Lemma 2.1 says that if $f$ is an $r$-dynamic $k$-coloring in $P_{n}$, then $k \geq 3$.

An observation in [9] stated that $\chi_{r} \geq \min \{\Delta(G, r)\}+1$ with equality holding when $G$ is a tree. Thus, when this observation is applied for paths of order $n \geq 3$, we have the following remark.

Remark 2.3. Let $P_{n}=\left(v_{1}, v_{2}, \ldots, v_{n}\right)$ be a path of order $n \geq 3$. If $r>1$, then $\chi_{r}\left(P_{n}\right)=3$.

The ideas presented in Lemma 2.1 and Remark 2.2 may be used to verify Remark 2.3 as follows. Define $f: V\left(P_{n}\right) \rightarrow\{1,2,3\}$ as follows

$$
f\left(v_{i}\right)= \begin{cases}1, & \text { if } i \equiv 1(\bmod 3) \\ 2, & \text { if } i \equiv 2(\bmod 3) \\ 3, & \text { if } i \equiv 0(\bmod 3)\end{cases}
$$

Then by Lemma $2.1 f$ is an $r$-dynamic 3-coloring of $P_{n}$. Hence, $\chi_{r}\left(P_{n}\right) \leq 3$. Now by Remark $2.2 \chi_{r}\left(P_{n}\right)=3$.

The next Lemma 2.4 characterizes an $r$-dynamic $k$-coloring in cycles. 
Lemma 2.4. Let $C_{n}=\left[v_{1}, v_{2}, \ldots, v_{n}\right]$ be a cycle of order $n \geq 3$. Then $f: V\left(C_{n}\right) \rightarrow\{1,2, \ldots, k\}$ is an $r$-dynamic $(r>1) k$-coloring of $C_{n}$ if and only if for all $i, f\left(v_{i-1}\right), f\left(v_{i}\right), f\left(v_{i+1}\right)$ are distinct.

Proof. Assume that $f$ is an $r$-dynamic $k$-coloring of $C_{n}$ and there exist $i$ such that at least two of $f\left(v_{i-1}\right), f\left(v_{i}\right), f\left(v_{i+1}\right)$ are not distinct. Consider the following cases: (Case 1. $\left.f\left(v_{i-1}\right)=f\left(v_{i}\right)\right)$ If $f\left(v_{i-1}\right)=f\left(v_{i}\right)$, then $f$ is not proper. This is a contradiction. (Case 2. $\left.f\left(v_{i-1}\right)=f\left(v_{i+1}\right)\right)$ If $f\left(v_{i-1}\right)=$ $f\left(v_{i+1}\right)$, then $f\left(N\left(v_{i}\right)\right)=f\left(\left\{v_{i-1}, v_{i+1}\right\}\right)=\left\{f\left(v_{i-1}\right)\right\}$, that is, $\left|f\left(N\left(v_{i}\right)\right)\right|=$ $\left|\left\{f\left(v_{i-1}\right)\right\}\right|=1<2=\min \{2, r\}$. This is a contradiction.

Conversely, assume that for all $i, f\left(v_{i-1}\right), f\left(v_{i}\right), f\left(v_{i+1}\right)$ are distinct. Let $v \in C_{n}$. Then $v=v_{i}$ for some $i$ and $\operatorname{deg}(v)=\operatorname{deg}\left(v_{i}\right)=2$. Thus, $|f(N(v))|=$ $\left|f\left(N\left(v_{i}\right)\right)\right|=\left|\left\{f\left(v_{i-1}\right), f\left(v_{i+1}\right)\right\}\right|=2=\min \{2, r\}$. This shows that $f$ is an $r$-dynamic $k$-coloring of $C_{n}$.

Remark 2.5. Lemma 2.4 says that if $f$ is an $r$-dynamic $k$-coloring in $C_{n}$, then $k \geq 3$.

Lemma 2.6. Let $G=(V, E)$ be a graph and $S=\left\{v_{1}, v_{2}, \ldots, v_{n}\right\} \subseteq V$ with $\left\langle\left\{v_{1}, v_{2}, \ldots, v_{n}\right\}\right\rangle=\left(v_{1}, v_{2}, \ldots, v_{n}\right)$. Let $f: V \rightarrow\{1,2,3\}$ be a vertex coloring of $G$. Then for all $i=2,3, \ldots, n-1, f\left(v_{i-1}\right), f\left(v_{i}\right), f\left(v_{i+1}\right)$ are distinct if and only if

$$
\left.f\right|_{S}\left(v_{j}\right)= \begin{cases}k_{1}, & \text { if } j \equiv 0(\bmod 3) \\ k_{2}, & \text { if } j \equiv 1(\bmod 3) \\ k_{3}, & \text { if } j \equiv 2(\bmod 3)\end{cases}
$$

where $k_{1} k_{2} k_{3}$ is a permutation of 1,2 and 3 .

Proof. Assume that $f\left(v_{i}\right), f\left(v_{i+1}\right)$ are distinct and without loss of generality

$$
\left.f\right|_{S}\left(v_{j}\right) \neq \begin{cases}1, & \text { if } j \equiv 0(\bmod 3) \\ 2, & \text { if } j \equiv 1(\bmod 3) \\ 3, & \text { if } j \equiv 2(\bmod 3)\end{cases}
$$

Since $f$ must be proper, there exists $i$ such that $\left.f\right|_{S}\left(v_{i}\right)=1,\left.f\right|_{S}\left(v_{i+1}\right)=2$, $\left.f\right|_{S}\left(v_{1+2}\right)=3$, and $\left.f\right|_{S}\left(v_{i+3}\right)=2$. This is a contradiction.

Conversely, assume that

$$
\left.f\right|_{S}\left(v_{j}\right)= \begin{cases}k_{1}, & \text { if } j \equiv 0(\bmod 3) \\ k_{2}, & \text { if } j \equiv 1(\bmod 3) \\ k_{3}, & \text { if } j \equiv 2(\bmod 3)\end{cases}
$$


where $k_{1} k_{2} k_{3}$ is a permutation of 123 , say without loss of generality

$$
\left.f\right|_{S}\left(v_{j}\right)= \begin{cases}1, & \text { if } j \equiv 0(\bmod 3) \\ 2, & \text { if } j \equiv 1(\bmod 3) \\ 3, & \text { if } j \equiv 2(\bmod 3)\end{cases}
$$

Let $j \in\{2,3, \ldots, n-1\}$, say without loss of generality $j \equiv 1(\bmod 3)$. Then $j-1 \equiv 0(\bmod 3)$ and $j+1 \equiv 2(\bmod 3)$. Hence, $f\left(v_{j-1}\right)=3, f\left(v_{j}\right)=1$, $f\left(v_{j+1}\right)=2$ are distinct.

A result in [10] stated that

$$
\chi_{2}\left(C_{n}\right)= \begin{cases}3, & \text { if } n \equiv 0(\bmod 3) \\ 5, & \text { if } n=5 \\ 4, & \text { otherwise }\end{cases}
$$

and an observation in [8] stated that if $r \geq \Delta(G)$, then $\chi_{r}(G)=\chi_{\Delta(G)}(G)$. By these and since $\Delta\left(C_{n}\right)=2$, we have the following remark.

Remark 2.7. Let $C_{n}=\left[v_{1}, v_{2}, \ldots, v_{n}\right]$ be a cycle of order $n \geq 3$. If $r>1$, then

$$
\chi_{r}\left(C_{n}\right)= \begin{cases}3, & \text { if } n \equiv 0(\bmod 3) \\ 5, & \text { if } n=5 \\ 4, & \text { otherwise }\end{cases}
$$

The ideas presented in Lemma 2.4, Remark 2.5 and Lemma 2.6 may be used to verify Remark 2.7 as follows. Let $C_{n}=\left[v_{1}, v_{2}, \ldots, v_{n}\right]$ be a cycle of order $n \geq 3$. Consider the following cases: (Case $1 . n \equiv 0(\bmod 3))$ If $n \equiv 0(\bmod 3)$, then we define $f: V\left(C_{n}\right) \rightarrow\{1,2,3\}$ as follows

$$
f\left(v_{i}\right)= \begin{cases}1, & \text { if } i \equiv 1(\bmod 3) \\ 2, & \text { if } i \equiv 2(\bmod 3) \\ 3, & \text { if } i \equiv 0(\bmod 3) .\end{cases}
$$

Then by Lemma $2.4 f$ is an $r$-dynamic 3-coloring of $C_{n}$. Thus, by Remark 2.5 $\chi_{r}\left(C_{n}\right)=3$.

$($ Case $2 . \quad n \equiv 1(\bmod 3))$ If $n \equiv 1(\bmod 3)$, then we define $f: V\left(C_{n}\right) \rightarrow$ $\{1,2,3,4\}$ as follows

$$
f\left(v_{i}\right)= \begin{cases}1, & \text { if } i \equiv 1(\bmod 3) \text { and } j \neq n \\ 2, & \text { if } i \equiv 2(\bmod 3) \\ 3, & \text { if } i \equiv 0(\bmod 3) \\ 4, & \text { if } i=n\end{cases}
$$


Then by Lemma $2.4 f$ is an $r$-dynamic 3-coloring of $C_{n}$. Hence, $\chi_{r}\left(C_{n}\right) \leq 4$. Suppose that $\chi_{r}\left(C_{n}\right)<4$, say without loss of generality $\chi_{r}\left(C_{n}\right)=3$. Let $f: V\left(C_{n}\right) \rightarrow\{1,2,3\}$ be an $r$-dynamic 3 -coloring of $C_{n}$. Then by Lemma 2.4 $f\left(v_{i-1}\right), f\left(v_{i}\right), f\left(v_{i+1}\right)$ are distinct for all $i=1,2, \ldots, n$. Let $S=\{1,2, \ldots$, $n-1\}$. Then $\langle S\rangle=(1,2, \ldots, n-1)$. By Lemma 2.6, without loss of generality

$$
f\left(v_{j}\right)= \begin{cases}1, & \text { if } j \equiv 0(\bmod 3) \\ 2, & \text { if } j \equiv 1(\bmod 3) \\ 3, & \text { if } j \equiv 2(\bmod 3)\end{cases}
$$

for all $i=1,2, \ldots, n-1$. Since there are only 3 colors, $f\left(v_{n}\right)$ is either 1,2 or 3 . Since $f$ must be a proper vertex coloring, $f\left(v_{n}\right)=2$. This implies that $f\left(v_{n-2}\right), f\left(v_{n-1}\right), f\left(v_{n}\right)$ are not distinct. This is a contradiction. Therefore, $\chi_{r}\left(C_{n}\right)=4$.

(Case $3 . n \equiv 2(\bmod 3)$ with $n \neq 5)$ If $n \equiv 1(\bmod 3)$ with $n \neq 5$, then we define $f: V\left(C_{n}\right) \rightarrow\{1,2,3,4\}$ as follows

$$
f\left(v_{i}\right)=\left\{\begin{array}{cl}
1, & \text { if } i \equiv 1(\bmod 4) \\
2, & \text { if } i \equiv 2(\bmod 4) \\
3, & \text { if } i \equiv 3(\bmod 4) \\
4, & \text { if } i \equiv 0(\bmod 4)
\end{array}\right.
$$

Then clearly $f$ is an $r$-dynamic 4-coloring of $C_{n}$. Hence, $\chi_{r}\left(C_{n}\right) \leq 4$. Suppose that $\chi_{r}\left(C_{n}\right)<4$, say $\chi_{r}\left(C_{n}\right)=3$. Using the same arguments in the proof of Case 2 , it can be shown also that $\chi_{r}\left(C_{n}\right)$ can not be less than 4 . Therefore, $\chi_{r}\left(C_{n}\right)=4$.

(Case 4. $n=5$ ) If $n=5$, then we define $f: V\left(C_{5}\right) \rightarrow\{1,2,3,4,5\}$ as follows $f\left(v_{i}\right)=i$. Then clearly $f$ is an $r$-dynamic 5-coloring of $C_{5}$. Hence, $\chi_{r}\left(C_{5}\right) \leq 5$. Suppose that $\chi_{r}\left(C_{5}\right)<5$, say $\chi_{r}\left(C_{5}\right)=4$. Then by Lemma 2.4 (without loss of generality) $f\left(v_{i}\right)=i$ for $i=1,2,3,4$. Since there are only 4 colors $f\left(v_{5}\right)$ is either 1 or 2 or 3 or 4 . This is a contradiction by Lemma 2.4 . Therefore, $\chi_{r}\left(C_{5}\right)=5$.

\section{3. r-Dynamic Chromatic Number of Complete Graphs and Empty Graphs}

This section presents the $r$-dynamic chromatic number of complete graphs and empty graphs. Theorem 3.1 characterizes graphs with $r$-dynamic chromatic number equal to its order when $r$ is less than the minimum degree $\delta$. 
Theorem 3.1. Let $G=(V, E)$ be graph of order $n \geq 3$, and $r \in \mathbb{N}$ with $r<\delta$. Then $\chi_{r}(G)=n$ if and only if $G \cong K_{n}$.

Proof. Assume that $\chi_{r}(G)=n$ and $G \not K_{n}$. If $G \not K_{n}$, then there exist $u, v \in V$ such that $u v \notin E$. Assume that $V=\left\{v_{1}, v_{2}, \ldots, v_{n-2}\right.$, $\left.v_{n-1}=u, v_{n}=v\right\}$. Define $f: V\left(K_{n}\right) \rightarrow\{1,2, \ldots, n-1\}$ as follows

$$
f\left(v_{i}\right)= \begin{cases}i, & \text { if } i \neq n \\ n-1, & \text { if } i=n\end{cases}
$$

Let $w \in V(G)$ and consider the following cases: (Case 1. $w=u$ or $w=$ $v$ ) Without loss of generality, if $w=u$, then $|f(N(w))|=\min \{r, \operatorname{deg}(u)\}$. (Case 2. $w \neq u$ and $w \neq v$ ) If $w \neq u$ and $w \neq v$, then $|f(N(w))| \geq r=$ $\min \{r, \operatorname{deg}(u)\}$ since $r<\delta$. Thus, $f$ is an $r$-dynamic $k$-coloring of $G$, that is, $\chi_{r}(G) \leq n-1$. This is a contradiction.

Conversely, assume that $G=K_{n}$. Then $n=\chi(G) \leq \chi_{r}(G) \leq n$. Hence, $\chi_{r}(G)=n$.

Theorem 3.2 characterizes graphs with $r$-dynamic chromatic number equal to 1 .

Theorem 3.2. Let $G=(V, E)$ be graph of order $n$, and $r \in \mathbb{N}$. Then $\chi_{r}(G)=1$ if and only if $G \cong \bar{K}_{n}$.

Proof. Assume that $\chi_{r}(G)=1$ and $G \nsubseteq \bar{K}_{n}$. If $G \nsubseteq K_{n}$, then there exist $u, v \in V$ such that $u v \in E$. Let $f: V\left(\bar{K}_{n}\right) \rightarrow\{1\}$ be an $r$-dynamic 1-coloring of $G$. Then $f(u)=1=f(v)$. This is a contradiction since $f$ must be proper.

Conversely, assume that $G=\bar{K}_{n}$. Define $f: V \rightarrow\{1\}$ by $f(v)=1$ for all $v \in V$. Let $u \in V$. Then $|f(N(u))|=|f(\varnothing)|=0=\min \{r, 0\}$. Clearly, $f$ is a proper coloring. Hence, $f$ is an $r$-dynamic 1-coloring of $G$. Accordingly, $\chi_{r}(G)=1$.

\section{2-Dynamic Chromatic Number of the Vertex Gluing of Graphs}

In this section we present the $r$-dynamic chromatic number of the vertex gluing of graphs. Lemma 4.1 characterizes an $r$-dynamic $k$-coloring in a graph.

Lemma 4.1. Let $G=(V, E)$ be graph and $f=\left\{V_{1}, V_{2}, \ldots, V_{k}\right\}$ be a $k$-coloring of $G$. Then $f$ is an $r$-dynamic $k$-coloring of $G$ if and only if

1. $\{u, v\} \nsubseteq V_{i}$ for all $i=1,2, \ldots, k$ whenever $u v \in E$, and 
2. there exist $\left\{q_{1}, q_{2}, \ldots, q_{\min \{r, \operatorname{deg}(v)\}}\right\} \subseteq\{1,2, \ldots, k\} \backslash\{i\}$ such that $N(v) \cap$ $V_{q_{j}} \neq \varnothing$ for all $j=1,2, \ldots, \min \{r, \operatorname{deg}(v)\}$ whenever $v \in V_{i}$ for some $i \in\{1,2, \ldots, k\}$.

Proof. Assume that $f=\left\{V_{1}, V_{2}, \ldots, V_{k}\right\}$ is an $r$-dynamic $k$-coloring of $G$. Let $u v \in E$. Since $f$ must be proper $u \in V_{s}$ and $v \in V_{t}$ for some $s, t \in$ $\{1,2, \ldots, k\}$ with $s \neq t$. Since $\left\{V_{1}, V_{2}, \ldots, V_{k}\right\}$ is a pairwise disjoint family, we must have $\{u, v\} \nsubseteq V_{i}$ for all $i=1,2, \ldots, k$. Next, let $v \in V_{i}$ for some $i \in\{1,2, \ldots, k\}$. Let $N(v)=\left\{v_{1}, v_{2}, \ldots, v_{m}\right\}$ where $m \geq \min \{r, \operatorname{deg}(v)\}$. Since $f$ is an $r$-dynamic $k$-coloring,

$$
\begin{aligned}
|f(N(v))|=\left|f\left(\left\{v_{1}, v_{2}, \ldots, v_{m}\right\}\right)\right|=\mid\left\{f\left(v_{1}\right), f\left(v_{2}\right), \ldots,\right. & \left.f\left(v_{m}\right)\right\} \mid \\
& \geq \min \{r, \operatorname{deg}(v)\} .
\end{aligned}
$$

This implies that there exist $\left\{q_{1}, q_{2}, \ldots, q_{\min \{r, \operatorname{deg}(v)\}}\right\} \subseteq\{1,2, \ldots, k\} \backslash\{i\}$ such that $v_{j} \in V_{q_{j}}$ for all $j=1,2, \ldots, \min \{r, \operatorname{deg}(v)\}$. Hence, there exist

$$
\left\{q_{1}, q_{2}, \ldots, q_{\min \{r, \operatorname{deg}(v)\}}\right\} \subseteq\{1,2, \ldots, k\} \backslash\{i\}
$$

such that $N(v) \cap V_{q_{j}} \neq \varnothing$ for all $j=1,2, \ldots, \min \{r, \operatorname{deg}(v)\}$.

Conversely, assume that conditions (1) and (2) hold. Let $u v \in E$. Then by (1), $\{u, v\} \nsubseteq V_{i}$ for all $i=1,2, \ldots, k$. Since $\left\{V_{1}, V_{2}, \ldots, V_{k}\right\}$ is a partition of $V$, $u \in V_{s}$ and $v \in V_{t}$ for some $s, t \in\{1,2, \ldots, k\}$ with $s \neq t$, that is, $f(u) \neq f(v)$. This shows that $f$ is proper. Next, let $v \in V$. Then $v \in V_{i}$ for some $i \in$ $\{1,2, \ldots, k\}$. By $(2)$, there exist $\left\{q_{1}, q_{2}, \ldots, q_{\min \{r, \operatorname{deg}(v)\}}\right\} \subseteq\{1,2, \ldots, k\} \backslash\{i\}$ such that $N(v) \cap V_{q_{j}} \neq \varnothing$ for all $j=1,2, \ldots, \min \{r, \operatorname{deg}(v)\}$. Thus, there exist $\left\{q_{1}, q_{2}, \ldots, q_{\min \{r, \operatorname{deg}(v)\}}\right\} \subseteq\{1,2, \ldots, k\} \backslash\{i\}$ such that $v_{j} \in V_{q_{j}}$ for all $j=$ $1,2, \ldots, \min \{r, \operatorname{deg}(v)\}$. This implies that if $N(v)=\left\{v_{1}, v_{2}, \ldots, v_{m}\right\}$ where $m \geq \min \{r, \operatorname{deg}(v)\}$, then $|f(N(v))|=\left|f\left(\left\{v_{1}, v_{2}, \ldots, v_{m}\right\}\right)\right|$ $=\left|\left\{f\left(v_{1}\right), f\left(v_{2}\right), \ldots, f\left(v_{m}\right)\right\}\right| \geq \min \{r, \operatorname{deg}(v)\}$.

Lemma 4.1, says that the colors of the color classes may be interchanged and the new coloring will still be an $r$-dynamic $k$-coloring.

Corollary 4.2. Let $G=(V, E)$ be a graph and $\left(V_{1}, V_{2}, \ldots, V_{k}\right)$ be an $r$-dynamic $k$-coloring of $G$. If $\left\{i_{1}, i_{2}, \ldots, i_{k}\right\}$ is a permutation of $\{1,2, \ldots, k\}$, then $\left(V_{i_{1}}, V_{i_{2}}, \ldots, V_{i_{k}}\right)$ is also an $r$-dynamic $k$-coloring of $G$.

Next, we have Lemma 4.3. This Lemma says that if $f$ is a proper coloring of $G$, then the restriction of $f$ to any subgraphs of $G$ is also a proper coloring.

Lemma 4.3. Let $G=(V, E)$ be graph and $H$ be a subgraph of $G$. If $f$ is a proper coloring of $G$, then $\left.f\right|_{V(H)}$ is a proper coloring of $H$. 
Proof. Let $f: V \rightarrow C$ be a proper coloring of $G$. If $f: V \rightarrow C$ be a proper coloring of $G$, then $f(u) \neq f(v)$ for all $u v \in E$. Let $a b \in E(H)$ and consider $\left.f\right|_{V(H)}$. If $a b \in E(H)$, then $a b \in E$. Hence, $f(a) \neq f(b)$, that is, $\left.f\right|_{V(H)}(a) \neq\left. f\right|_{V(H)}(b)$. This shows that $\left.f\right|_{V(H)}$ is a proper coloring of $H$.

Next, we have Lemma 4.3. The Lemma says that if $f$ is an $r$-dynamic $k$ coloring of $G$, then the restriction of $f$ to the vertex set of any subgraph $H$ of $G$ is an $r$-dynamic $k$-coloring of $H$.

Lemma 4.4. Let $G=(V, E)$ be graph and $H$ be a subgraph of $G$. If $f$ is an $r$-dynamic $k$-coloring of $G$, then $\left.f\right|_{V(H)}$ is an $r$-dynamic $k$-coloring of $H$.

Proof. Let $f: V \rightarrow C$ be an $r$-dynamic $k$-coloring. If $f: V \rightarrow C$ is an $r$-dynamic $k$-coloring of $G$, then $f$ is a proper coloring, and for all vertices $v$ in $V,|f(N(v))| \geq \min \left\{r, \operatorname{deg}_{G}(v)\right\}$. By Lemma $\left.4.3 f\right|_{V(H)}$ is a proper vertex coloring of $H$. Now, let $v \in V(H)$. Then

$$
\begin{aligned}
& \left|f\left(N_{G}(v)\right)\right|-\left|f\left(N_{H}(v)\right)\right| \leq\left|N_{G}(v)\right|-\left|N_{H}(v)\right| \\
& \Rightarrow\left|f\left(N_{G}(v)\right)\right|-\left|f\left(N_{H}(v)\right)\right| \leq \operatorname{deg}_{G}(v)-\operatorname{deg}_{H}(v) \\
& \Rightarrow \quad\left|f\left(N_{G}(v)\right)\right|-\operatorname{deg}_{G}(v) \leq\left|f\left(N_{H}(v)\right)\right|-\operatorname{deg}_{H}(v) \\
& \Rightarrow \quad 0 \leq\left|f\left(N_{H}(v)\right)\right|-\operatorname{deg}_{H}(v) \\
& \Rightarrow \quad \operatorname{deg}_{H}(v) \leq\left|f\left(N_{H}(v)\right)\right| \\
& \Rightarrow \quad \min \left\{r, \operatorname{deg}_{H}(v)\right\} \leq\left|f\left(N_{H}(v)\right)\right| \text {. }
\end{aligned}
$$

This shows that $\left.f\right|_{V(H)}$ is an $r$-dynamic $k$-coloring of $H$.

We call Corollary 4.5 the monotonicity property.

Corollary 4.5. Let $G=(V, E)$ be graph and $H$ be a subgraph of $G$. Then $\chi_{r}(G) \geq \chi_{r}(H)$.

Proof. Let $\mathscr{F}=\{f: f$ is an $r$-dynamic $k$-coloring of $G\}$ and $\mathscr{G}=\{g: g$ is an $r$-dynamic $k$-coloring of $H\}$. Then by Lemma $4.4, \mathscr{I}=\left\{\left.f\right|_{V(H)}: f \in \mathscr{F}\right\}$ is a subset of $\mathscr{G}$. Next, let $\mathscr{A}=\left\{|f|_{V(H)}(V(H)) \mid\right.$ : $\left.\left.f\right|_{V(H)} \in \mathscr{I}\right\}$ and $\mathscr{B}=\{|g(V(H))|: g \in \mathscr{G}\}$. Then, $\mathscr{A} \subseteq \mathscr{B}$. Thus, $\chi_{r}(G) \geq$ $\chi_{r}(H)$ since $|f(V(H))| \leq|f(V)|$ for all $f \in \mathscr{F}$.

Theorem 4.6. Let $G_{1}$ and $G_{2}$ be connected graphs with $\chi_{r}\left(G_{1}\right) \geq 3$. Let $G$ be the graph obtained by identifying a vertex in $G_{1}$ and a vertex in $G_{2}$, that is $G$ is a vertex gluing of $G_{1}$ and $G_{2}$. If $r=2$, then $\chi_{r}(G)=\max \left\{\chi_{r}\left(G_{1}\right), \chi_{r}\left(G_{2}\right)\right\}$.

Proof. Let $G_{1}=\left(V_{1}, E_{1}\right)$ and $G_{2}=\left(V_{2}, E_{2}\right)$ be a graphs. Let $G$ be the graph obtained by identifying $u \in V_{1}$ and $v \in V_{2}$, that is $G$ is a vertex gluing 
of $G_{1}$ and $G_{2}$. Let $f_{1}=\left(V_{1}^{(1)}, V_{2}^{(1)}, \ldots, V_{k_{1}}^{(1)}\right)$ and $f_{2}=\left(V_{1}^{(2)}, V_{2}^{(2)}, \ldots, V_{k_{2}}^{(2)}\right)$ be $r$-dynamic $k_{1}$-coloring of $G_{1}$ and $r$-dynamic $k_{2}$-coloring of $G_{2}$, respectively, such that $k_{1}=\chi_{r}\left(G_{1}\right)$ and $k_{2}=\chi_{r}\left(G_{2}\right)$. Consider the following cases: (Case 1. $\operatorname{deg}_{G_{1}}(u)=1$ or $\operatorname{deg}_{G_{2}}(v)=1$ ) Without loss of generality assume that $\operatorname{deg}_{G_{1}}(u)=1$. Let $w$ be a neighborhood of $u$ in $G_{1}$ and $z$ be a neighborhood of $v$ in $G_{2}$. Without loss of generality, assume that $u \in V_{1}^{(1)}, w \in V_{2}^{(1)}, v \in V_{s}^{(2)}$ and $z \in V_{t}^{(2)}$. Then by Corollary $4.2, f_{3}=\left(V_{i_{1}}^{(2)}, V_{i_{2}}^{(2)}, \ldots, V_{i_{k_{2}}}^{(2)}\right)$ with

$$
i_{m}= \begin{cases}1 & \text { if } m=s \\ s & \text { if } m=1 \\ 3 & \text { if } m=t \\ t & \text { if } m=3 \\ m & \text { otherwise }\end{cases}
$$

is also an $r$-dynamic $k$-coloring of $G_{2}$. Now, let $f: V(G) \rightarrow$ $\left\{1,2, \ldots, \max \left\{\chi_{2}\left(G_{1}\right), \chi_{2}\left(G_{2}\right)\right\}\right\}$ be given by

$$
f(w)= \begin{cases}f_{1}(w) & \text { if } w \in V_{1} \\ f_{3}(w) & \text { if } w \in V_{2}\end{cases}
$$

that is,

$$
\begin{aligned}
f= & \left(V_{j_{1}}, V_{j_{2}}, V_{j_{3}}, \ldots, V_{j_{k_{1}}}\right) \\
= & \left(V_{1}^{(1)} \cup V_{i_{s}}^{(2)}, V_{2}^{(1)} \cup V_{i_{2}}^{(2)}, V_{3}^{(1)} \cup V_{i_{t}}^{(2)}, \ldots, V_{k_{2}}^{(1)} \cup V_{i_{k_{2}}}^{(2)}, V_{k_{2}+1}^{(1)},\right. \\
& \left.\ldots, V_{k_{1}}^{(1)}\right)
\end{aligned}
$$

(without loss of generality we assume here that $k_{1} \geq k_{2}$ ). Let $x y \in E(G)$. Then either $x y \in E_{1}$ or $x y \in E_{2}$. Without loss of generality we assume that $x y \in E_{1}$. Since $f_{1}=\left(V_{1}^{(1)}, V_{2}^{(1)}, \ldots, V_{k_{1}}^{(1)}\right)$ is an $r$-dynamic $k$-coloring, by Lemma 4.1 , $\{x, y\} \nsubseteq V_{i}^{(1)}$ for all $i=1,2, \ldots, k_{1}$. Since $x y \in E_{1}, x, y \in V_{1}$. Hence, there exists $s, t \in\left\{1,2, \ldots, k_{1}\right\}$ with $s \neq t$ such that $x \in V_{s}$ and $y \in V_{t}$, that is, $x \in V_{j_{s}}$ and $y \in V_{j_{t}}$. Since is $\left\{V_{j_{1}}, V_{j_{2}}, V_{j_{3}}, \ldots, V_{j_{k_{1}}}\right\}$ a pairwise disjoint family, we have $\{x, y\} \nsubseteq V_{j_{i}}$ for all $i=1,2, \ldots, k_{1}$.

Next, let $y \in V(G)$. Consider the following subcases: (Subcase 1. either $y \in V_{1} \backslash V_{2}$ or $\left.y \in V_{2} \backslash V_{1}\right)$. Without loss of generality, assume that $y \in V_{1} \backslash V_{2}$, say $y \in V_{j_{r}}$. Then since $f_{1}$ is an $r$-dynamic $k$-coloring, by Lemma 4.1 there exist $s, t \in\left\{1,2, \ldots, k_{1}\right\} \backslash\{r\}$ such that $V_{j_{s}}^{(1)} \cap N_{G_{1}}(y) \neq \varnothing$ and $V_{j_{t}}^{(1)} \cap N_{G_{1}}(y) \neq \varnothing$. This implies that there exist $s, t \in\left\{1,2, \ldots, k_{1}\right\} \backslash\{r\}$ such that $V_{j_{s}} \cap N_{G}(y) \neq \varnothing$ and $V_{j_{t}} \cap N_{G}(y) \neq \varnothing$. 
(Subcase 2. $y \in V_{1} \cap V_{2}$ ) If $y \in V_{1} \cap V_{2}$, then $y=v=u$. Since $w \in V_{2}^{(1)} \subseteq V_{j_{2}}$ and $z \in V_{3}^{(2)}$, we have $V_{j_{2}} \cap N_{G}(y) \neq \varnothing$ and $V_{j_{3}} \cap N_{G}(y) \neq \varnothing$.

(Case 2. $\operatorname{deg}_{G_{1}}(u)>1$ or $\operatorname{deg}_{G_{2}}(v)>1$ ) Without loss of generality, assume that $u \in V_{1}^{(1)}$ and $v \in V_{2}^{(2)}$. Then by Corollary $4.2, f_{3}=\left(V_{i_{1}}^{(2)}, V_{i_{2}}^{(2)}, \ldots, V_{i_{k_{2}}}^{(2)}\right)$ with

$$
i_{m}= \begin{cases}1 & \text { if } m=2 \\ 2 & \text { if } m=1 \\ m & \text { otherwise }\end{cases}
$$

is also an 2-dynamic $k$-coloring of $G_{2}$. Now, let $f: V(G) \rightarrow$ $\left\{1,2, \ldots, \max \left\{\chi_{2}\left(G_{1}\right), \chi_{2}\left(G_{2}\right)\right\}\right\}$ be given by

$$
f(w)= \begin{cases}f_{1}(w) & \text { if } w \in V_{1} \\ f_{3}(w) & \text { if } w \in V_{2},\end{cases}
$$

that is,

$$
\begin{aligned}
f & =\left(V_{j_{1}}, V_{j_{2}}, V_{j_{3}}, \ldots, V_{j_{k_{1}}}\right) \\
& =\left(V_{1}^{(1)} \cup V_{i_{1}}^{(2)}, V_{2}^{(1)} \cup V_{i_{2}}^{(2)}, \ldots, V_{k_{2}}^{(1)} \cup V_{i_{k_{2}}}^{(2)}, V_{k_{2}+1}^{(1)}, \ldots, V_{k_{1}}^{(1)}\right)
\end{aligned}
$$

(without loss of generality we assume here that $k_{1} \geq k_{2}$ ). Let $x y \in E(G)$. Then either $x y \in E_{1}$ or $x y \in E_{2}$. Without loss of generality we assume that $x y \in E_{1}$. Since $f_{1}=\left(V_{1}^{(1)}, V_{2}^{(1)}, \ldots, V_{k_{1}}^{(1)}\right)$ is an $r$-dynamic $k$-coloring, by Lemma 4.1 , $\{x, y\} \nsubseteq V_{i}^{(1)}$ for all $i=1,2, \ldots, k_{1}$. Since $x y \in E_{1}, x, y \in V_{1}$. Hence, there exists $s, t \in\left\{1,2, \ldots, k_{1}\right\}$ with $s \neq t$ such that $x \in V_{s}$ and $y \in V_{t}$, that is, $x \in V_{j_{s}}$ and $y \in V_{j_{t}}$. Since is $\left\{V_{j_{1}}, V_{j_{2}}, V_{j_{3}}, \ldots, V_{j_{k_{1}}}\right\}$ a pairwise disjoint family, we have $\{x, y\} \nsubseteq V_{j_{i}}$ for all $i=1,2, \ldots, k_{1}$.

Next, let $y \in V(G)$. Without loss of generality, assume that $y \in V_{1}$, say $y \in V_{r}^{(1)}$, that is $y \in V_{j_{r}}$. Then since $f_{1}$ is an $r$-dynamic $k$-coloring, by Lemma 4.1 there exist $s, t \in\left\{1,2, \ldots, k_{1}\right\} \backslash\{r\}$ such that $V_{s}^{(1)} \cap N_{G_{1}}(y) \neq \varnothing$ and $V_{t}^{(1)} \cap N_{G_{1}}(y) \neq \varnothing$. This implies that there exist $s, t \in\left\{1,2, \ldots, k_{1}\right\} \backslash\{r\}$ such that $V_{j_{s}} \cap N_{G}(y) \neq \varnothing$ and $V_{j_{t}} \cap N_{G}(y) \neq \varnothing$.

Accordingly, by Lemma $4.1 f$ is a 2 -dynamic $k$-coloring. Therefore, $\chi_{2}(G) \leq$ $k_{1}=\max \left\{\chi_{r}\left(G_{1}\right), \chi_{r}\left(G_{2}\right)\right\}$.

Suppose that $\chi_{2}(G)<k_{1}=\max \left\{\chi_{r}\left(G_{1}\right), \chi_{r}\left(G_{2}\right)\right\}$. Note that $G_{1}$ is a subgraph of $G$. Thus by Corollary $4.5, \chi_{2}\left(G_{1}\right) \leq \chi_{2}(G)$. This is a contradiction.

Theorem 4.6 may be extended to the vertex gluing of a finite number of graphs. 
Corollary 4.7. Let $n \in \mathbb{N}$ and $G_{1}, G_{2}, \ldots, G_{n}$ be graphs. Let $G$ be the vertex gluing of $G_{1}, G_{2}, \ldots, G_{n}$. If $r=2$, then

$$
\chi_{2}(G)=\max \left\{\chi_{r}\left(G_{1}\right), \chi_{r}\left(G_{2}\right), \ldots, \chi_{r}\left(G_{n}\right)\right\}
$$

Proof. This follows from Theorem 4.6 by induction.

\section{5. r-Dynamic Chromatic Number of Forest}

This section presents the $r$-dynamic chromatic number of forest. We note that a forest is a finite union of trees, and a tree is a finite vertex gluing of paths. The next remark gives the 2-dynamic chromatic number of trees. An observation in [8] states that $\chi_{r}(G) \geq \min \{\Delta(G), r\}+1$ where equality holds when $G$ is a tree. The next remark verified this idea for $r=2$.

Remark 5.1. Let $T$ be a tree of order $n \geq 3$. If $r=2$, then $\chi_{r}(T)=3$.

Proof. Assume that $T$ is the vertex gluing of $n$ paths $P_{1}, P_{2}, \ldots, P_{n}$. Then by Corollary 4.7,

$$
\chi_{2}(T)=\max \left\{\chi_{r}\left(P_{1}\right), \chi_{r}\left(P_{2}\right), \ldots, \chi_{r}\left(P_{n}\right)\right\}=3 .
$$

The next result gives the $r$-dynamic chromatic number of the union of two graphs.

Theorem 5.2. Let $G$ and $H$ be graphs. Then

$$
\chi_{r}(G \cup H)=\max \left\{\chi_{r}(G), \chi_{r}(H)\right\} .
$$

Proof. Let

$$
f_{1}: V(G) \rightarrow\left\{1,2, \ldots, \chi_{r}(G)\right\} \text { and } f_{1}: V(G) \rightarrow\left\{1,2, \ldots, \chi_{r}(H)\right\}
$$

be $r$-dynamic $k$-colorings of $G$ and $H$, respectively. Define $f: V(G \cup H) \rightarrow$ $\left\{1,2, \ldots, \max \left\{\chi_{r}(G), \chi_{r}(H)\right\}\right\}$ by

$$
f(v)= \begin{cases}f_{1}(v) & \text { if } v \in V(G) \\ f_{2}(v) & \text { if } v \in V(H) .\end{cases}
$$


(Claim 1. $f$ is a proper vertex coloring) Let $u v \in E(G \cup H)$. Then either $u v \in E(G)$ or $u v \in E(H)$. Without loss of generality, assume that $u v \in E(G)$. If $u v \in E(G)$, then since $f_{1}$ is proper, $f_{1}(u) \neq f_{1}(v)$, that is, $f(u) \neq f(v)$. This shows the claim.

(Claim 2. $|f(N(v))| \geq \min \left\{r, \operatorname{deg}_{G \cup H}(v)\right\}$ for all $v \in V(G \cup H)$ ) Let $v \in V(G \cup H)$. Then either $v \in V(G)$ or $v \in V(H)$. Without loss of generality, assume that $v \in V(G)$. If $v \in V(G)$, then since $f_{1}$ is an $r$-dynamic $k$-coloring, $\left|f_{1}(N(v))\right| \geq \min \left\{r, \operatorname{deg}_{G}(v)\right\}$, that is, $|f(N(v))| \geq \min \left\{r, \operatorname{deg}_{G \cup H}(v)\right\}$. This shows the claim.

By Claims 1 and 2, $\chi_{r}(G \cup H) \leq \max \left\{\chi_{r}(G), \chi_{r}(H)\right\}$. And by Corollary 4.5, $\chi_{r}(G \cup H)=\max \left\{\chi_{r}(G), \chi_{r}(H)\right\}$.

Theorem 5.2 may be extended to finite union of graphs.

Corollary 5.3. Let $n \in \mathbb{N}$ and $G_{1}, G_{2}, \ldots, G_{n}$ be graphs. Then

$$
\chi_{r}\left(G_{1} \cup G_{2} \cup \cdots \cup G_{n}\right)=\max \left\{\chi_{r}\left(G_{1}\right), \chi_{r}\left(G_{2}\right), \ldots, \chi_{r}\left(G_{n}\right)\right\} .
$$

We revisit an observation in [8] which states that $\chi_{r}(G) \geq \min \{\Delta(G)$, $r\}+1$ where equality holds when $G$ is a tree. Since a forest is a finite union of trees, the next result gives the $r$-dynamic chromatic number of forest.

Corollary 5.4. Let $F$ be a forest with a component of order $n \geq 3$. Then $\chi_{r}(F)=\min \{\Delta(G), r\}+1$.

Proof. Assume that $F$ is the union of $n$ trees $T_{1}, T_{2}, \ldots, T_{n}$. Then by Corollary 5.3,

$$
\chi_{r}(F)=\max \left\{\chi_{r}\left(T_{1}\right), \chi_{r}\left(T_{2}\right), \ldots, \chi_{r}\left(T_{n}\right)\right\}=\min \{\Delta(G), r\}+1 .
$$

\section{References}

[1] H.J. Lai, B. Montgomery, H. Poon, Upper bounds of dynamic chromatic number, Ars Combin. 68 (2003) 193-201.

[2] A. Taherkhani, $r$-Dynamic Chromatic Number of Graphs, arXiv:1401.6470v1 [math.CO] 24 Jan 2014.

[3] B. Montgomery, Dynamic Coloring of Graphs (Ph.D Dissertation), West Virginia University, 2001. 
[4] B. Gao, L. Sun, H. Sung, H.J. Lai, On the Difference Between Dynamic Chromatic and Chromatic Number of Graphs, Journal of Mathematical Sciences: Advances and Applications 34 (2015), 1-10.

[5] Y. Chen, S. Fan, H.-J. Lai, H. Song, L. Sun, On dynamic coloring for planar graphs and graphs of higher genus, Discrete Applied Mathematics 160 (2012), 1064-1071.

[6] Y. Kim, S.J. Lee, S.-I. Oum, Dynamic Coloring of Graphs Having No $K_{5}$ Minor, arXiv:1201.2142v3 [math.CO] 15 March 2015.

[7] M. Alishahi, On the dynamic coloring of graphs, Discrete Applied Mathematics 159 (2011) 152-156.

[8] R. Kang, T. Muller, D.B. West, On $r$-dynamic coloring of grids, Discrete Applied Mathematics 186 (2015) 286-290.

[9] S. Jahanbekam, J. Kim, Suil O, D.B. West, On $r$-dynamic coloring of graphs, submitted for publication.

[10] H.-J. Lai, B. Montgomery, Dynamic coloring of graphs, West Virginia University, 2002.

[11] Wikipedia contributors. "Graph coloring." Wikipedia, The Free Encyclopedia. Wikipedia, The Free Encyclopedia, 30 Jun. 2016. Web. 8 Jul. 2016.

[12] J. Gross, J. Yellen, Handbook of Graph Theory, CRC Press LLC, N. W., Corporate Blvd, Boca Raton, Florida, 2000. 